\title{
The un-unified airway: two distinct TLR-mediated pathways regulate house dust mite-induced allergic disease in the upper and lower airways
}

\author{
Joo-Heon Yoon \\ Department of Otorhinolaryngology, Yonsei University College of Medicine, Seoul, Korea
}

\begin{abstract}
Allergic rhinitis (AR) and allergic asthma (AA) are main allergic diseases in the upper and lower airways, and allergic symptoms of both AR and AA are caused by aberrant T helper type 2 (TH2) responses initiated by innate immune responses to allergen exposure. However, in spite of different immunologic condition at the nasal and lung mucosal surface, distinctive regulatory mechanism of early immune responses to environmental allergens in each of their mucosal epithelia are totally unknown. Here, we demonstrate that HDM-derived $\beta$-glucans are the main pathogen associated molecular pattern (PAMP) molecules responsible for triggering AR and this was dependent on a TLR 2-mediated innate immunity in nasal mucosa. We also show that allergic features of HDM-induced AR were not dependent on LPS-TLR4 signaling pathway, although HDM-induced AA was dependent on LPS-TLR4 signaling pathway but not $\beta$-glucan-TLR2 signaling pathways in our study. This data provide a novel concept that definitely different mucosal immune systems exist in the nose and lung epithelium in response to allergen exposure and this distinctive innate immunity play an essential role for triggering AR or AA in the upper and lower airways, respectively.
\end{abstract}

